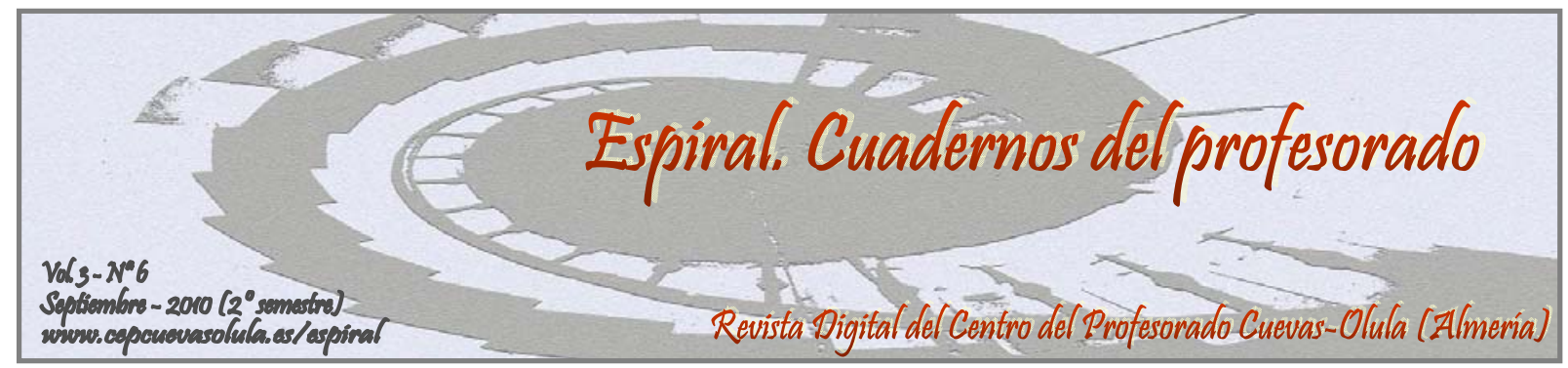

\title{
EL AGRUPAMIENTO FLEXIBLE: UN MARCO ORGANIZATIVO COMO MEDIO PARA LA ATENCIÓN A LA DIVERSIDAD EN LA EDUCACIÓN SECUNDARIA OBLIGATORIA
}

\section{THE FLEXIBLE GROUPING: AN ORGANIZATIONAL FRAMEWORK AS A MEANS FOR THE ATTENTION TO DIVERSITY IN COMPULSORY SECONDARY EDUCATION}

\author{
María José Ramos Alcázar ${ }^{(1)}$ y Francisco José Martínez López ${ }^{(2)}$ \\ ${ }^{(1)}$ IES Pueblos de la Villa, Fuente Álamo, Murcia, España \\ ${ }^{(2)}$ IES Ricardo Ortega, Fuente Álamo, Murcia, España
}

RESUMEN: En este trabajo presentamos la experiencia llevada a cabo con el agrupamiento flexible del aula como marco organizativo y metodológico, desarrollado en alumnado de ESO. Surge como respuesta a unas necesidades educativas ocasionadas por la diversidad de éste en nuestras aulas. Analizamos cómo plantear la organización de espacios, qué circunstancias han de darse, considerando aquellos condicionantes que deben contribuir favorablemente a que desarrollemos con éxito esta metodología. Se pretende alcanzar unos efectos positivos en la actitud y motivación del alumnado que ha de traducirse además en una mejora de los rendimientos académicos.

Palabras clave: agrupamiento flexible, atención a la diversidad, aula, metodología.

ABSTRACT: In this work we show the experience carried out with the flexible grouping of the classroom as organizational and methodological frame, developed in students of Secondary Education. It arises as response to many educational needs caused by the diversity of these students in our classrooms. We analyze how to plan the organization of spaces, what circumstances have to be given, considering those determining factors that contribute favourably to the development successful of this methodology. We try to reach great number of positive effects in the attitude and motivation of the students that have to turn in addition in an improvement of the academic performances.

Key words: flexible grouping, attention to the diversity, classroom, methodology.

Ramos Alcázar, M.J. \& Martínez López, F.J. (2010). El agrupamiento flexible: un marco organizativo como medio para la atención a la diversidad en la Educación Secundaria Obligatoria. Espiral. Cuadernos del Profesorado [en línea], 3(6), 65-72. Disponible en: http://www.cepcuevasolula.es/espiral.

Fecha de recepción: 21/12/2009

Fecha de aceptación: 07/07/2010
Enviar correspondencia a: mariajose.ramos@murciaeduca.es 


\section{1.- INTRODUCCIÓN. ORIGEN DE LA EXPERIENCIA.}

En las últimas décadas el tratamiento de la diversidad está adquiriendo, en el contexto educativo, un protagonismo cada vez mayor, siendo entendida la diversidad como la expresión plural de las diferencias y traducida como el reconocimiento y la aceptación del otro como parte de una misma entidad colectiva que nos incluye (Vilar García, 2009).

Nos hallamos ante una sociedad inmersa en la globalidad, donde además, los movimientos migratorios, como un elemento más de ese fenómeno, nos afectan e involucran a todos. La sociedad en general y nuestro entorno más inmediato en particular, nuestros centros docentes, en definitiva, no son una excepción. Por tanto, nuestras aulas, donde focalizaremos este trabajo, que tampoco son ajenas a este escenario, nos evidencian irremediablemente esta diversidad.

Se ofrece el diseño meditado de la organización escolar y las estrategias metodológicas como herramientas de trabajo para hacer frente a una respuesta adecuada a nuestros alumnos y alumnas. Como señala Sánchez Hipona (1994) cuando argumenta desde la perspectiva de análisis de la organización escolar y de la metodología didáctica:

“... la respuesta educativa a la diversidad plantea al profesorado y a la escuela la necesidad de considerar estrategias organizativas y didácticas para abordar necesidades e intereses diferentes y peculiares intensidades de esfuerzos requeridos para cada alumno y alumna en concreto" (p. 106).

La experiencia desarrollada, parte desde la iniciativa de un grupo de profesores y profesoras del IES Ricardo Ortega y posteriormente en el IESO Pueblos de la Villa de Fuente Álamo de Murcia, donde se continuará con esta metodología, ahora más consolidada y depurada, después de haber sido iniciada durante el curso 2005/06.

\section{2.- LA DIVERSIDAD EN EL ALUMNADO.}

Con el fuerte incremento de la llegada de población inmigrante extranjera, entra en escena la diversidad cultural y de etnia (García, Granados y García-Cano, 2000). Avanzamos en una cultura de globalización que, por tanto, deriva en que personas muy diferentes en capacidades, costumbres, ideas... deben convivir en un mismo espacio, también el que ofrece el centro educativo.

Estas últimas décadas han puesto de manifiesto una realidad muy variada, diversa y un tanto compleja, cuya intensificación en los últimos años ha suscitado la necesidad de "hacer algo”. Hay que establecer nuevas respuestas, más conscientes, encaminadas y dirigidas a afrontar una realidad educativa un tanto diferente y algo o bastante desajustada, que además es asociada al concepto de diferencia cultural, convertida ésta en panacea de la desigualdad, aunque podríamos estar ante un desplazamiento y ocultación de realidades de la desigualdad social como señala Carrasco (2003).

Sin embargo, otros autores parten de que la diversidad y la desigualdad en el aula no provienen de la pertenencia a grupos de diferente cultura, raciales o de nacionalidad distinta, sino que son fruto de la coexistencia no reconocida de diferentes formas de interaccionar en el aula, como señalan Márquez Lepe y García-Cano Torrico (2004), cuando trabajan esta propuesta teórica desde su proyecto sobre Aprendizaje Multicultural e Instructivo con Grupos Heterogéneos en el Aula (AMIGHA), versión castellana de Cooperative Learning in European Contexts (CLIEC) enmarcado dentro del programa europeo Comenius.

El conjunto de la población que se encuentra escolarizada en las etapas de educación obligatoria exige disponer de una capacidad de respuesta a nuestro sistema educativo para atender a la diversidad que nuestro alumnado plantea ante su educación, de manera que se logren de la mejor forma posible los objetivos establecidos en el mismo, además de que todos puedan desarrollar al máximo sus capacidades.

El planteamiento sistémico de la diversidad, debe abordarse desde un enfoque institucional, teniendo en cuenta la atención del alumnado con sus diversas cualidades, y atendiendo a sus situaciones, tanto de índole individual como social, que plantean diferencias que deben ser atendidas desde puntos de vista organizativo y curricular, como señala Casanova (2003), en cuanto a sus características generales:

- Estilos cognitivos.

- Ritmos de aprendizaje diferentes.

- Distintas motivaciones.

- Intereses diversos. 
También en cuanto a las diferencia de capacidades:

- Altas capacidades.

- Capacidades medias.

- Bajas capacidades.

Así como diferencias sociales:

- Desconocimiento importante de la lengua vehicular del sistema.

- Pertenencia a entornos sociales desfavorecidos

- Minorías étnicas.

- Itinerancias.

Podemos determinar, por tanto, que entendiendo el currículo en su sentido más amplio y comprensivo, debe dar respuesta organizativa a la forma de abordar los contenidos de enseñanza y del aprendizaje, ante una sociedad, antes "normal", ahora entendida como "diversa”.

Hemos de ajustar la oferta educativa, para dar una respuesta más certera y aproximada que permita que todos los alumnos desarrollen las mismas capacidades en la medida de sus verdaderas posibilidades, proporcionándoles el tipo de intervención que precisen. Como señala Lozano Martínez y García Nadal (1999), el objetivo fundamental es lograr la mayor participación posible de estos alumnos y alumnas en el currículo ordinario, atendiendo a la vez a las necesidades específicas e individuales de los mismos.

\section{3.- EL AGRUPAMIENTO FLEXIBLE COMO MARCO ORGANIZATIVO. OBJETIVOS A CONSEGUIR.}

Al igual que Sancho Gil (1991), entendemos la concepción y distribución del espacio escolar como la expresión de un modo de entender el proceso de enseñanza-aprendizaje. En este sentido, encaminamos el diseño del aula flexible, entendiéndolo además como una respuesta a la diversidad del alumnado, que debe hacerse desde la consideración de una apuesta clara y precisa, tanto desde el centro educativo, con su equipo de profesores al frente, como de la propia administración educativa. Hemos de asumir plenamente que se trata de ofrecer la posibilidad de trabajar curricularmente, adaptándose a unas necesidades educativas más ajustadas a la realidad del alumnado.

Pretendemos alcanzar una serie de objetivos que repercutirán directamente en el propio discente, además de en el centro educativo en el que este marco organizativo se ponga en funcionamiento.

Destacamos los siguientes:

- Posibilitar la atención a la riqueza diversa que ocupa nuestras aulas, de manera más centrada y próxima a sus verdaderos intereses.

- Garantizar los progresos de nuestro alumnado de acuerdo con las capacidades y posibilidades de cada individuo, desarrollando una estrategia metodológica basada en el aprendizaje significativo.

- Conseguir mejores resultados académicos del alumnado, en general, paliando, en parte, el desfase curricular, por un lado, y fomentando la adecuada progresión y el desarrollo curricular óptimo por otro.

- Disminuir considerablemente aquellos casos de disrupción que en circunstancias standards dificultarían el desarrollo de las clases.

- Favorecer el desarrollo de la identidad personal en condiciones de equidad y calidad.

- Reconocer el abanico de posibilidades organizativas de las aulas con agrupamientos flexibles como estrategia metodológica eficaz para el tratamiento de la diversidad.

En definitiva, mejorar los rendimientos del alumnado, afectando no sólo a las notas, que Rodríguez Espinar (1982) las considera como la referencia de los resultados escolares y como una realidad de que se nos impone sobre cualquier otra, sino además al grado de satisfacción psicológica y de bienestar. De este modo también lo hará el clima de relaciones y el tono afectivo en torno a la tarea escolar, que adquirirá dimensiones más humanizadas y optimistas (Adell i Cueva, 2002).

\section{4.- ASPECTOS METODOLÓGICOS.}

La principal finalidad que pretendemos obtener con los agrupamientos flexibles es el de aproximar por semejanza en los niveles de competencia curricular al alumnado más heterogéneo y diverso entre sí que nos podamos encontrar, y en cualquier área del currículo. Con esta agrupación se consigue homogeneizar al grupo de alumnos y alumnas y atender así a sus distintos intereses y conocimientos. Por otra 
parte, este rediseño de los espacios induce además al comportamiento del alumnado afectado e incide en la dinámica de la comunicación y de la convivencia, al hallarse todos ellos interrelacionados (Contreras, 1990).

Para atender a la diversidad, arrancamos de una estrategia metodológica basada en los principios del aprendizaje significativo, como señala Sánchez Delgado (2002), bien atendido y más ajustado, en tanto que partimos desde sus verdaderos niveles de conocimientos, para empezar a construir y a crecer en una adquisición de contenidos más equilibrada. Pero no basta, hemos de recurrir a reconfigurar la distribución grupal, flexibilizando para homogeneizar intereses y competencias del alumnado.

¿Cómo hemos de configurar los agrupamientos flexibles? En primer lugar, es primordial conocer a todo el alumnado que va a ser susceptible de tal aplicación. Para ello, una evaluación previa o inicial es imprescindible. Además, al igual que otros autores (Keeves, 1972; Pérez-Serrano, 1981; Rodríguez Espinar, 1982; Gascó Molina, 1984; Adell i Cueva, 1995) consideramos las calificaciones escolares del curso anterior como uno de los indicadores fiables del rendimiento del curso siguiente, siendo las materias instrumentales las mejores predictoras del rendimiento global, como también afirma Pérez Serrano (1981).

De su análisis determinamos qué tipo de alumnado tenemos, qué condiciones presenta, peculiaridades, niveles de competencia curricular, diversidad... En definitiva, toda la información necesaria para que podamos poner en funcionamiento el proceso de agrupación más idónea en cada caso.

La distribución de cada uno de ellos en el nivel que responda a sus necesidades, hemos de realizarla en función del número de niveles que el centro nos permita organizar. El número de horas asignado y el de profesores disponibles para tal fin, dependerán en última instancia de la disponibilidad que el centro asigne y a su vez a éste, le haya asignado la administración educativa pertinente. No obstante, manejando los mínimos que la normativa permite, es decir, invirtiendo las horas de apoyos y desdobles que se asignan a cada caso, podemos empezar a diseñar estos agrupamientos.

Nos centraremos entre tres, incluso pudiéndose dar el caso de cuatro niveles, respondiendo cada uno a los siguientes perfiles de alumnado:

a) Nivel 1: alumnos y alumnas con altos niveles de competencia curricular. Todos aquellos que pueden seguir el currículo ordinario sin ningún problema e incluso destacan satisfactoriamente.

b) Nivel 2: atendería al alumnado con bajo nivel de rendimiento y ciertas dificultades en el aprendizaje. Podrían seguir el currículo ordinario, pero con ligeras adaptaciones en los contenidos y con una metodología de trabajo muy específica.

c) Nivel 3: Representaría al grupo de alumnos y alumnas con graves dificultades en el aprendizaje y con un acusado desfase curricular debido a cualquier situación o problemática que lo haya avocado ante tal estado (disruptivos, absentistas, minorías, etc.). Incluso alumnos y alumnas con adaptaciones curriculares significativas.

En aquellos casos en los que, tanto las características del propio alumnado como los medios humanos lo permitieran, podríamos establecer otro nivel de agrupamiento más.

d) Nivel 4: nos ayudaría a definir aún más la situación de alumnos entre los niveles 2 y 3 , puesto que generalmente la realidad pone de manifiesto que podemos encontrar un grupo intermedio entre ambos, con un perfil de alumnos y alumnas con dificultades más acusadas que los del nivel 2, pero no tan extremas como las que nos encontramos con aquellos del nivel 3. De esta forma conseguimos alcanzar una mayor aproximación a la realidad del grupo, lo que redundará en una atención más personalizada y ajustada a sus verdaderas necesidades educativas.

Hemos de ser realistas ante la organización en cuatro niveles. Se trata de una disposición organizativa que, en mayor grado, se enfrenta a problemas de recursos humanos, y por ende, económicos. Estamos, en este caso, ante un escenario que en cuanto a su puesta en práctica es un tanto improbable. Hemos de tener en cuenta que tanto el centro como la administración educativa tienen sus propias limitaciones.

Añadir además que, este diseño de niveles trae consigo la simultaneidad del profesorado de la materia en la que se pone en práctica este sistema organizativo. 
El siguiente esquema vale de representación gráfica como quedan organizados: dos grupos tienen que coincidir (simultanear) en las sesiones de la misma materia sujeta a nuestro estudio del agrupamiento flexible. Pongamos el ejemplo de que tal situación se produce con los grupos A y B.

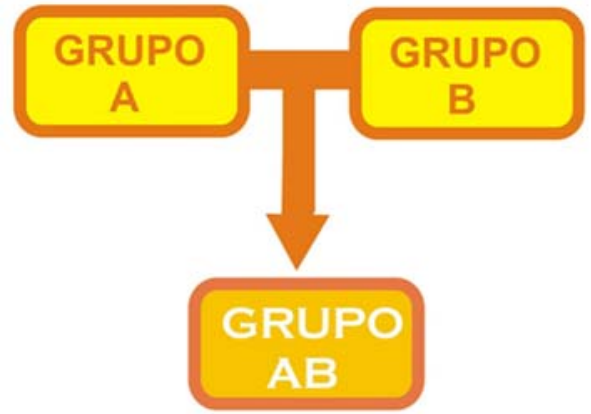

Figura 1.- Situación previa. Consideración de un único grupo $\mathrm{AB}$.

De éstos establecemos la consideración de un gran grupo, único grupo al que llamaremos $\mathrm{AB}$ (figura 1) y sobre el que estableceremos la distribución por niveles. Para el caso de tres niveles se requerirán simultáneamente tres profesores dando clase.

La distribución del alumnado sería, una vez evaluado previamente a cada uno de los alumnos y alumnas de los dos grupos originales, se procede a la distribución de cada uno de ellos de acuerdo al nivel de competencia que se ajuste mejor al nivel establecido (figura 2). Por lo que quedará repartido dicho alumnado entre tres niveles de competencia, tres grupos de la materia sujeta a nuestro estudio, que responderá a sus necesidades, demandas o carencias.

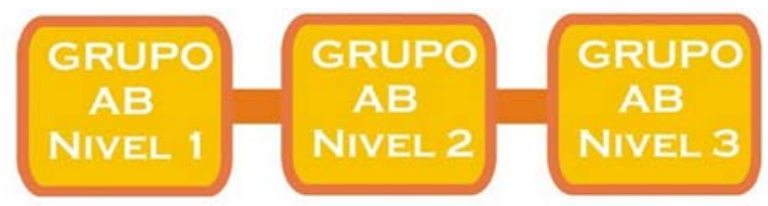

Figura 2.- Situación final. Agrupamientos en 3 niveles.

De esta manera, se diluye el grupo clase que de un principio vienen configurados, ya que dejan de ser alumnos y alumnas del grupo de referencia A o B, pasando a ser del nivel 1, 2 ó 3. De igual modo ocurre con el profesorado, ya no es el profesor de un grupo A o B, sino que se corresponde y relaciona con uno de los 3 niveles.

Este procedimiento que se podría realizan en cualquier momento del curso, obviamente, procede ser desarrollado al principio del mismo.
Es aquí cuando lógicamente responde a su razón de ser, atendiendo a la organización anticipada de inicio de curso.

Por otra parte, este tipo de marco metodológico ofrece una mayor recursividad a la hora de afrontar situaciones posteriores, una vez iniciado el curso. En aquellos momentos en los que se incorporan alumnos nuevos, alumnos inmigrantes o itinerantes, por ejemplo, que llegan en cualquier momento del curso, el proceso se repetiría tal cual hemos expuesto. De esta forma también se afianza y asegura una atención a este tipo de diversidad, de modo más eficaz y ajustado.

Una vez que tenemos la infraestructura adecuada, tres niveles por ejemplo, y al alumnado perfectamente determinado a cual de los niveles le correspondería estar, es el momento de iniciar el proceso.

En ese momento, con todo el proceso metodológico iniciado puede ocurrir que se detecte el caso de algún alumno que no esté adecuadamente ubicado. Confirmado y valorado por el equipo docente al frente, hace los reajustes pertinentes, reubicando a dicho alumno en el nivel correcto. Para ello, la coordinación entre los miembros de ese equipo docente ha de ser total y continua, mínimo una vez a la semana, podría ser el espacio temporal requerido.

De este modo, lo que se detecte se subsana inmediatamente con el fin de no "perjudicar" a nadie. Estos cambios o reajustes siempre atienden a las demandas y criterio del profesorado y casi nunca a las de los alumnos, padres u otros miembros de la comunidad educativa. Se escucharán posibles sugerencias, aunque la valoración última y el criterio firme parte del equipo de profesores y profesoras responsable. Se tratará de realizar el menor número posible de dichos cambios a lo largo del curso, porque repercutiría gravemente en el alumnado. Estos cambios quedarían dentro de excepcionalidades y no procesos habituales.

Es muy poco probable que alumnos de un nivel inferior, promocionen o cambien a un nivel superior; dentro del mismo curso escolar. No obstante, las excepcionalidades siempre existen y hay que tenerlas previstas.

Sin embargo, de un año para otro, sí suele suceder con mayor frecuencia el que alumnos que han estado durante un curso en uno de los niveles, cuando se vuelvan a reconfigurar los agrupamientos de éstos, "promocionen" a otro 
nivel superior, o por el contrario, desciendan a otro inferior.

\section{5.- ORGANIZACIÓN DEL PROFESORADO. REPERCUSIONES.}

Para poner todo este marco metodológico en funcionamiento y que verdaderamente se lleguen a conseguir los objetivos pretendidos, tenemos que contar con la pieza básica del mismo: el equipo docente. Dependerá de él buena parte, tanto de los logros y los éxitos, como de los fracasos. Si tenemos en cuenta propuestas como las de Santos Guerra (1999), apoyadas por Núñez Gómez y Cerrillo Martín (2004), el profesorado debe tener flexibilidad para el cambio, permeabilidad con el medio, racionalidad en el funcionamiento y flexibilidad sobre la acción, todo ello pasado por el tamiz de sus emociones y de sus exigencias éticas, sociales y políticas. Sin embargo, como señala Pérez Ferra (2000:212), en la misma línea que De Vicente (2000), "el profesorado que se toma en serio su actividad profesional suele mantener una agonía constante entre sus creencias más profundas, sobre lo que considera que es la educación y la realidad diaria que le dice cotidianamente que ha de cambiar".

El equipo de profesores que formen parte de esta organización, debe estar en verdadera consonancia y plenamente integrado. Son los auténticos conductores de esta ardua e interesante empresa. Si se da el caso de falta de interés, bajo convencimiento, poca implicación, o cualquier otro factor que no apoye o contribuya al buen funcionamiento del sistema, todo fallará. Si por el contrario, el compromiso, la participación y la implicación son elevadas, estaremos contribuyendo muy favorablemente a la consecución de logros y éxitos importantes.

Es por ello, por lo que en muchos de los casos en que se propone esta metodología de trabajo, no se consiguen los resultados esperados. La mayoría de las veces, por la falta de motivación de una parte del profesorado implicado. Cuando uno o algunos de los miembros del equipo no participen plenamente, la organización se verá afectada y como consecuencia, los resultados pretendidos no se alcanzarán satisfactoriamente. De ahí la importancia que en este marco organizativo tiene su plena intervención e implicación.

Por otra parte, tomando como referencia los niveles de competencia que se establezcan, así será la organización del equipo docente que esté al frente de la misma.

Cuando se establecen los niveles se precisará de al menos un docente para cada uno de ellos, y por tanto uno más que grupos se pretendan flexibilizar. Tal y como se refleja en el siguiente esquema (figura 3):

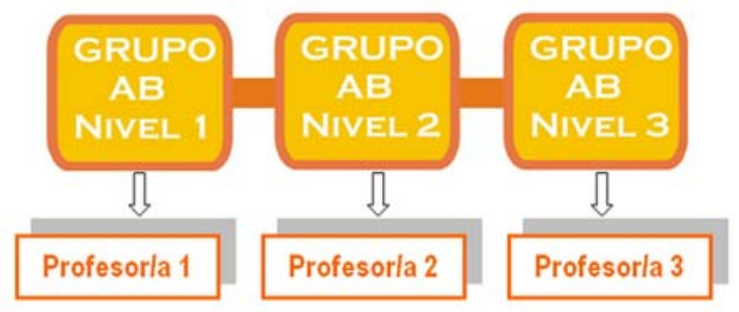

Figura 3.- Vínculo entre dos grupos/tres niveles y profesorado.

Se han flexibilizado dos grupos (A y B), obteniendo como resultado tres niveles y por tanto, tres profesores a su cargo, uno para cada uno de ellos (figura 3).

En el caso en que se agrupen tres grupos para establecer cuatro niveles, corresponderían cuatro profesores. En definitiva, la estructuración y organización de dichos agrupamientos dependerá en tal medida de las posibilidades del centro y recursos humanos disponibles.

En el peor de los casos, se podría dar la situación de flexibilizar los grupos entre sí, obteniendo el mismo número de niveles, bastando para ello los profesores al frente de cada uno de los grupos (figura 4). De este modo, no se extrae ningún nivel más, que surgiría como mínimo, al homogeneizar los distintos grupos a flexibilizar.

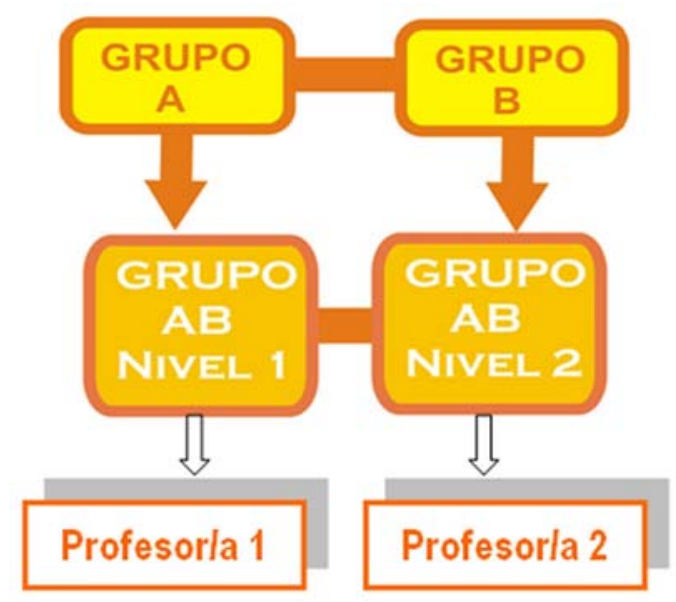

Figura 4.- Vínculo entre dos grupos/dos niveles y profesorado. 
No olvidemos que como condición fundamental para poder flexibilizar los grupos, es necesario que estén simultaneando sus sesiones para poder aplicar este proceso.

Como consecuencia de esta nueva estructuración, el profesorado implicado en estos agrupamientos estará muy coordinado entre sí en sus tareas. Además, se simultanearán en las funciones y labores que recaigan en los grupos de alumnos, tales como reuniones de equipo educativo, sesiones de evaluación, coordinación de actividades de cualquier tipo, porque todos tendrán alumnado de todos los grupos y repercutirán en todos esas tareas. De alguna manera, podríamos decir que duplica sus funciones propias de docente desde el ámbito de su área al tratar a dos grupos de alumnos y alumnas, aunque en realidad se trate de uno sólo.

Además de todo ello, este profesorado al frente, tiene más complicado desarrollar funciones de tutor del grupo original (previo a la flexibilización) que le sea asignado, porque no tendrá a todos los tutorandos y puede darse el caso de no conocerlos desde su área.

Pero a pesar de estos posibles inconvenientes, las ventajas siempre son mayores $\mathrm{y}$ merecedoras de esta pequeña sobrecarga, porque permiten alcanzar los objetivos perseguidos a través de esta metodología organizativa.

\section{6.- CONCLUSIONES.}

La búsqueda de criterios para la adecuada distribución del espacio escolar del aula adaptado al alumnado es una tarea del equipo docente, que ha de ser contemplada en el Proyecto Educativo de Centro y debe contar con la experiencia del profesorado implicado en el curso anterior.

Además de los consiguientes modificaciones en la configuración del espacio y en la organización, los agrupamientos flexibles van a afectar la interrelación entre el alumnado y los docentes, que establecerán los modos de aprender y de enseñar, al igual que determinarán la configuración de cómo se experimentará en el aula el conocimiento académico.

Se trata de una propuesta metodológica, como estrategia didáctica, que va a exigir un mayor esfuerzo y responsabilidad por parte del alumnado, pero también un mayor control y exigencia por parte del profesorado implicado.

A pesar de todas las ofertas y posibles soluciones que nos ayudarán a paliar las carencias, dificultades y limitaciones que el planteamiento del agrupamiento flexible como metodología nos ofrece, afirmamos que éste es una opción de carácter endógeno, es decir, que debe de gestarse, evolucionar y partir desde el convencimiento del propio actor que la va a llevar a cabo, y éste no es otro que el docente. De no ser así, todos los compromisos y esfuerzos que se requieran para desarrollar esta estrategia metodológica, se enfrentarán a constantes problemas y trabas, que dificultarán su ejecución y sobre todo la obtención de unos resultados satisfactorios.

Se afronta la diversidad desde un amplio abanico de perspectivas, además de la estrictamente curricular y académica. Como ya se ha reflejado, el alumnado queda atendido de acuerdo a las demandas y exigencias que cada uno presente, consiguiendo tanto las carencias detectadas, como ofrecer mayores posibilidades y atención a quienes ampliamente alcancen los objetivos previstos.

En definitiva, estamos ante una estrategia metodológica en consonancia con el verdadero aprendizaje significativo, que precisa de un trabajo cooperativo y de una actitud reflexiva, abierta, dinámica y exigente, por parte del profesorado.

\section{7.- REFERENCIAS BIBLIOGRÁFICAS.}

Adell i Cueva, M.A. (1995). ¿Están satisfechos los escolares con sus notas? Revista Escuela Española, 3219, 131-132.

Adell i Cueva, M.A. (2002). Estrategias para mejorar el rendimiento académico de los adolescentes. Madrid: Psicología Pirámide.

Carrasco, S. (2003). Antropología de la Educación y Antropología para la Educación. En A. González \& J.L. Molina (Eds.). Abriendo surcos sobre la tierra: Homenaje a Ramón Valdés del Toro. Barcelona: Publicacions d'Antropología Cultural UAB.

Casanova, M.A. (2003). El tratamiento de la diversidad en la educación básica española. Revista Iberoamericana de Educación, 31, 121143.

Contreras, J. (1990). Enseñanza, Curriculum y Profesorado. Madrid: Akal.

De Vicente, P.S. de (2000). Un profesor poderoso para una nueva escuela. En M. Pérez \& J.A. Torres (Coords.), La calidad de los procesos de enseñanza (pp. 237-268). Barcelona: Oikos-Tay.

García, F.J., Granados, A. \& García-Cano, M. (2000). Interculturalidad y Educación en la década de 
los noventa: un análisis crítico. Sevilla: Consejería de Educación y Ciencia.

Gasco Molina, J. (1984). Predicción Multivariada de rendimiento escolar en el ciclo inicial de EGB. Valencia: Ciencias de la Educación.

Lozano Martínez, J. \& García Nadal, R. (1999). Adaptaciones curriculares para la diversidad. Murcia: KR.

Márquez Lepe, E. \& García-Cano Torrico, M. (2004). Debates sobre educación intercultural desde su práctica en el aula. Portularia, 4, 143-152.

Núñez Gómez, E. \& Cerrillo Martín, M.R. (2004). Aulas de Enlace: una iniciativa pedagógica, integradora e intercultural. Tendencias Pedagógicas 9, 203-215.

Pérez Ferra, M. (2000). La escuela como organización que aprende. Enseñanza, 17-18, 201-216.

Pérez Serrano, G. (1981). Origen social y rendimiento escolar. Madrid: Centro de Investigaciones Sociológicas (CIS).
Keeves, J.P. (1972). Educational environment and student attainment. Estocolmo: Almquist and Wiksell.

Rodríguez Espinar, S. (1982). Factores de rendimiento escolar. Barcelona: Oikus-Tau.

Sánchez Delgado, P. (2002). Atención a la diversidad cultural en los centros educativos. Revista Complutense de Educación, 13 (2), 677-706.

Sánchez Hipona, M.P. (1994). La organización y el espacio escolar en el marco de la integración. Revista Complutense de Educación, 5 (2), 105120.

Sancho Gil, J.M. (1991). El entorno físico y simbólico de la enseñanza. Cuadernos de Pedagogía, 192, 73-77.

Santos Guerra, M.A. (1999). Hoy toca teoría. Revista de Innovación Curricular, 80, 87-88.

Vilar García, M. (2009). La diversidad cultural en educación desde enfoques multi e interculturales: conceptos y realidades. Sociedad y Discurso, 16, 102-118.

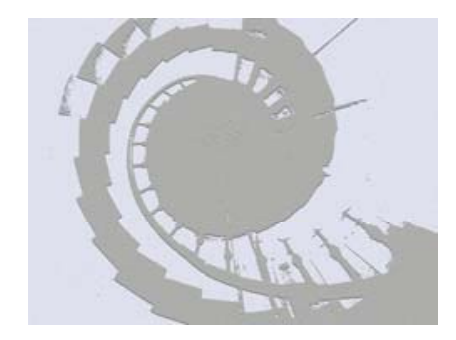

